\title{
Incomparable, non-isomorphic and minimal Banach spaces
}

\author{
by
}

Christian Rosendal (Pasadena, CA)

\begin{abstract}
A Banach space contains either a minimal subspace or a continuum of incomparable subspaces. General structure results for analytic equivalence relations are applied in the context of Banach spaces to show that if $E_{0}$ does not reduce to isomorphism of the subspaces of a space, in particular, if the subspaces of the space admit a classification up to isomorphism by real numbers, then any subspace with an unconditional basis is isomorphic to its square and hyperplanes, and the unconditional basis has an isomorphically homogeneous subsequence.
\end{abstract}

1. Introduction. This paper contains results in the intersection of the geometry of Banach spaces and descriptive set theory. The general problem of our study is a generalisation of the homogeneous space problem. Namely, what can be said about a Banach space with "few" non-isomorphic subspaces? In particular, will such a space necessarily satisfy more regularity properties than a general space? Will it necessarily have subspaces of a given type?

The paper is divided into two parts, of which the first contains a proof of the following:

Theorem 1. Let $X$ be an infinite-dimensional Banach space. Then $X$ contains either a minimal subspace or a continuum of pairwise incomparable subspaces.

Recall that two spaces are said to be incomparable if neither of them embed into the other, and a space is minimal if it embeds into all of its infinite-dimensional subspaces and is itself infinite-dimensional. Therefore, if a space is saturated with pairs of incomparable subspaces, it has a continuum of incomparable subspaces.

The homogeneous space problem, which was solved in the positive by the combined efforts of Gowers [10] and Komorowski and Tomczak-Jaegermann [18], is the problem of whether any infinite-dimensional space, isomorphic to all its infinite-dimensional subspaces, must necessarily be isomorphic to $\ell_{2}$.

2000 Mathematics Subject Classification: Primary 46B03; Secondary 03E15. 
As a continuation of this one can ask how many isomorphism classes of subspaces a non-Hilbertian space has to contain. Infinitely many? A continuum? Even for some of the classical spaces this question is still open, though recently progress has been made by Ferenczi and Galego [7].

Our theorem and proof turn out to have something to say about the following two problems of Gowers ([10, Problems 7.9 and 7.10]):

- Determine which partial orders can be realised as the set of subspaces of an infinite-dimensional Banach space under the relation of embeddability. Or at least find strong conditions such a partial order must necessarily satisfy.

- Find further applications of the main determinacy result in [10]. In particular, are there any applications that need its full strength, i.e., that need it to hold for analytic and not just open sets?

Our Theorem 1 says that any such partial order must have either a minimal element or an antichain of continuum size. And, as will be evident, the proof does in fact very much need the full strength of the determinacy result.

We mention that our proof relies heavily on methods of logic and we have therefore included a short review of the most basic notions of set theory indispensable to understand the proof. Also for the benefit of the non-analyst we recall some standard notions from Banach space theory.

Before presenting the results of the second part, we will first need this brief review.

1.1. Descriptive set theory. Our general reference for descriptive set theory will be the book [17] by Kechris, whose notation will be adopted here.

A Polish space is a separable completely metrisable space. A measurable space whose algebra of measurable sets are the Borel sets of some Polish topology is said to be standard Borel. These spaces turn out to be completely classified up to Borel isomorphism by their cardinality, which can be either countable or equal to that of the continuum. A subset of a standard Borel space is analytic if it is the image by a Borel function of some standard Borel space, and coanalytic if its complement is analytic. It is $C$-measurable if it belongs to the smallest $\sigma$-algebra containing the Borel sets and closed under the Suslin operation. In particular, analytic sets are $C$-measurable as they can be obtained by the Suslin operation applied to a sequence of Borel sets. $C$-measurable sets in Polish spaces satisfy most of the classical regularity properties, such as universal measurability and the Baire property. We denote by $\boldsymbol{\Sigma}_{1}^{1}, \boldsymbol{\Pi}_{1}^{1}$ and $\boldsymbol{\Sigma}_{2}^{1}$ the classes of analytic, coanalytic and Borel images of coanalytic sets respectively. A classical result of Sierpiński states that any $\boldsymbol{\Sigma}_{2}^{1}$ set is the union of $\aleph_{1}$ Borel sets. 
Let $X$ be a Polish space and $\mathcal{F}(X)$ denote the set of closed subsets of $X$. We endow $\mathcal{F}(X)$ with the following $\sigma$-algebra that renders it a standard Borel space. The generators are the following sets, where $U$ varies over the open subsets of $X$ :

$$
\{F \in \mathcal{F}(X) \mid F \cap U \neq \emptyset\} .
$$

The resulting measurable space is called the Effros-Borel space of $X$.

Fix some basis $\left\{U_{n}\right\}$ for the space $C\left(2^{\mathbb{N}}\right)$ and define the Borel set $\mathfrak{B}$ by

$$
\begin{aligned}
\mathfrak{B}=\left\{F \in \mathcal{F}\left(C\left(2^{\mathbb{N}}\right)\right) \mid \forall n\left(0 \in U_{n} \rightarrow F \cap U_{n} \neq \emptyset\right) \wedge \forall n, m, l \forall r, t \in \mathbb{Q}\right. \\
\left.\left(r U_{n}+t U_{m} \subseteq U_{l} \wedge F \cap U_{n} \neq \emptyset \wedge F \cap U_{m} \neq \emptyset \rightarrow F \cap U_{l} \neq \emptyset\right)\right\} .
\end{aligned}
$$

This evidently consists of all the closed linear subspaces of $C\left(2^{\mathbb{N}}\right)$ and, as $C\left(2^{\mathbb{N}}\right)$ is isometrically universal for separable Banach spaces, any separable Banach space has an isometric copy in $\mathfrak{B}$. We can therefore view $\mathfrak{B}$ as the standard Borel space of all separable Banach spaces. When one wants to restrict attention to the subspaces of some particular space $X$ one only needs to consider the Borel subset $\{Y \in \mathfrak{B} \mid Y \subseteq X\}$. Moreover, it is not hard to see that most reasonably definable properties and relations are at most $\boldsymbol{\Sigma}_{2}^{1}$ in $\mathfrak{B}$ or $\mathfrak{B}^{n}$; for example, the relations of isometry and isomorphism are both analytic in $\mathfrak{B}^{2}$, exactly as expected. For a systematic study of the descriptive complexity of different relations between Banach spaces, one should mention the article [3] by B. Bossard.

A theme of descriptive set theory that has been extensively developed the last fifteen years or so, is the Borel reducibility ordering of analytic equivalence relations on standard Borel spaces. This ordering is defined as follows: Suppose $\boldsymbol{E} \subseteq X^{2}$ and $\boldsymbol{F} \subseteq Y^{2}$ are analytic equivalence relations on standard Borel spaces $X$ and $Y$. We say that $\boldsymbol{E}$ is Borel reducible to $F$, in symbols $\boldsymbol{E} \leq_{\mathrm{B}} \boldsymbol{F}$, if there is a Borel measurable function $f: X \rightarrow Y$ such that for all $x, y \in X$,

$$
x \boldsymbol{E} y \Leftrightarrow f(x) \boldsymbol{F} f(y) .
$$

Moreover, when $X$ and $Y$ are Polish and $f$ can be taken to be continuous, we write $\boldsymbol{E} \leq_{\mathrm{c}} \boldsymbol{F}$.

Heuristically, $X$ represents a class of mathematical objects (e.g., separable Banach spaces) that we wish to classify up to $\boldsymbol{E}$-equivalence (e.g., isomorphism) by complete invariants belonging to some other category of mathematical objects. A reduction $f: X \rightarrow Y$ of $\boldsymbol{E}$ to $\boldsymbol{F}$ then corresponds to a classification of $X$-objects up to $\boldsymbol{E}$-equivalence by $Y$-objects up to $\boldsymbol{F}$-equivalence.

Another way of viewing the Borel reducibility ordering is as a refinement of the concept of cardinality. It provides a concept of relative cardinality for quotient spaces in the absence of the axiom of choice, since a reduction of $\boldsymbol{E}$ 
to $\boldsymbol{F}$ is essentially an injection of $X / \boldsymbol{E}$ into $Y / \boldsymbol{F}$ admitting a Borel lifting from $X$ to $Y$.

A few words on the power of the continuum: We say that an analytic equivalence relation $\boldsymbol{E}$ on a standard Borel space $X$ has a continuum of classes if there is an uncountable Borel set $B \subseteq X$ consisting of pairwise $\boldsymbol{E}$ inequivalent points. This is known to be stronger than just demanding that there should be some bijection between the set of classes and $\mathbb{R}$. There are for example analytic equivalence relations having exactly $\aleph_{1}$ classes, but not having a continuum of classes (in the above sense) in any model of set theory. But an uncountable Borel set is always Borel isomorphic to $\mathbb{R}$, independently of the size of the continuum. For an example of an analytic equivalence relation with uncountably many but not continuum many classes, define $\boldsymbol{E}$ on $\mathcal{P}(\mathbb{Q})$ by

$A \boldsymbol{E} B \Leftrightarrow\left[\left(A,<_{\mathbb{Q}}\right)\right.$ and $\left(B,<_{\mathbb{Q}}\right)$ are not wellordered or $\left.\left(A,<_{\mathbb{Q}}\right) \cong\left(B,<_{\mathbb{Q}}\right)\right]$.

If $A$ is some infinite subset of $\mathbb{N}$, we denote by $[A]^{\mathbb{N}}$ the space of all infinite subsets of $A$ equipped with the topology induced by the product topology on $2^{A}$. Furthermore, for two sets $A$ and $B$ we write $A \subseteq^{*} B$ iff $A \backslash B$ is finite. Then $A \subsetneq^{*} B$ iff $A \subseteq^{*} B$ but $B \nsubseteq^{*} A$. Also, when $A \subseteq \mathbb{N}$ and $k \in \mathbb{N}$ we let $A / k=\{n \in A \mid n>k\}$. We will occasionally also consider natural numbers as ordinals, so that $n=\{0,1, \ldots, n-1\}$.

We will repeatedly use the following result of Ellentuck and Louveau extending results of Galvin-Prikry for Borel sets and of Silver for analytic sets: if $\mathcal{A} \subseteq[\mathbb{N}]^{\mathbb{N}}$ is a $C$-measurable set, then there is some $A \in[\mathbb{N}]^{\mathbb{N}}$ with either $[A]^{\mathbb{N}} \subseteq \mathcal{A}$ or $[A]^{\mathbb{N}} \cap \mathcal{A}=\emptyset$.

This has the consequence that if $f:[\mathbb{N}]^{\mathbb{N}} \rightarrow X$ is some $C$-measurable function with values in some Polish space $X$, then there is some $A \in[\mathbb{N}]^{\mathbb{N}}$ such that the restriction of $f$ to $[A]^{\mathbb{N}}$ is continuous.

Among the simpler analytic equivalence relations are those that admit a classification by real numbers, i.e., those that are Borel reducible to the identity relation on $\mathbb{R}$. These are said to be smooth. It turns out that among Borel equivalence relations there is a minimum, with respect to $\leq_{\mathrm{B}}$, nonsmooth one, which we denote by $E_{0}$ (see [13]). It is defined on $[\mathbb{N}]^{\mathbb{N}}$ as the relation of eventual agreement, i.e.,

$$
A E_{0} B \Leftrightarrow \exists n A / n=B / n .
$$

To see that $E_{0}$ is non-smooth, suppose towards a contradiction that $f$ : $[\mathbb{N}]^{\mathbb{N}} \rightarrow \mathbb{R}$ is a Borel function such that $A E_{0} B \Leftrightarrow f(A)=f(B)$. Then there is some infinite $C \subseteq \mathbb{N}$ such that the restriction of $f$ to $[C]^{\mathbb{N}}$ is continuous. But, as the equivalence class of $C$ is dense in $[C]^{\mathbb{N}}$, this means that $f$ is constant on $[C]^{\mathbb{N}}$, contradicting the fact that $[C]^{\mathbb{N}}$ intersects more than one equivalence class. 
On the other hand, any uncountable Borel set $\mathcal{B} \subseteq[\mathbb{N}]^{\mathbb{N}}$ of pairwise almost disjoint sets will witness that $E_{0}$ has a continuum of classes.

From this it follows that any analytic equivalence relation to which $E_{0}$ reduces has a continuum of classes, but does not admit a classification by real numbers.

After these preliminary remarks we can state our second result.

TheOREM 2. Let $X$ be a Banach space with an unconditional basis $\left(e_{n}\right)$. If $E_{0}$ does not Borel reduce to isomorphism between subspaces generated by subsequences of the basis (and in particular if these admit a classification by real numbers), then any space spanned by a subsequence is isomorphic to its square and hyperplanes. Furthermore, in this case there is a subsequence of the basis such that all of its subsequences span isomorphic spaces.

For example, as the usual basis of Tsirelson's space does not have a subsequence all of whose subsequences span isomorphic spaces, this shows that there is no isomorphic classification of the subspaces of Tsirelson's space by real numbers.

This result can be coupled with Gowers's dichotomy [10] proving:

Theorem 3. Let $X$ be a separable Banach space. Either $E_{0}$ Borel reduces to isomorphism between its subspaces, or $X$ contains a reflexive subspace with an unconditional basis all of whose subsequences span isomorphic spaces.

For the above we will need some Ramsey type results for product spaces and some constructions for reducing $E_{0}$. These results seem to have an independent interest apart from their applications to Banach space theory in that they classify minimal counterexamples to Ramsey properties in product spaces. For $\boldsymbol{E}$ an equivalence relation on $[\mathbb{N}]^{\mathbb{N}}$ and $A \in[\mathbb{N}]^{\mathbb{N}}$, we say that $A$ is $\boldsymbol{E}$-homogeneous if $A \boldsymbol{E} B$ for all $B \in[A]^{\mathbb{N}}$. Now we can state another of our results:

THEOREM 4. Let $\boldsymbol{E}$ be an analytic equivalence relation on $[\mathbb{N}]^{\mathbb{N}}$ invariant under finite changes. Either $E_{0}$ Borel reduces to $\boldsymbol{E}$, or $\boldsymbol{E}$ admits a homogeneous set.

1.2. Schauder bases. Let $X$ be some separable Banach space and $\left(e_{i}\right)$ a non-zero sequence in $X$. We say that $\left(e_{i}\right)$ is a basis for $X$ if any vector $x$ in $X$ can be uniquely written as a norm convergent series $x=\sum a_{i} e_{i}$. In that case, the biorthogonal functionals $e_{k}^{*}\left(\sum a_{i} e_{i}\right):=a_{k}$ and the projections $P_{n}\left(\sum a_{i} e_{i}\right):=\sum_{i=0}^{n} a_{i} e_{i}$ are in fact continuous and moreover their norms are uniformly bounded.

If $\left(e_{i}\right)$ is some non-zero sequence that is a basis for its closed linear span, written $\left[e_{i}\right]$, we say that it is a basic sequence in $X$. The property of $\left(e_{i}\right)$ 
being a basic sequence can also be equivalently stated as the existence of a constant $K \geq 1$ such that for any $n \leq m$ and $a_{0}, a_{1}, \ldots, a_{m} \in \mathbb{R}$,

$$
\left\|\sum_{i=0}^{n} a_{i} e_{i}\right\| \leq K\left\|\sum_{i=0}^{m} a_{i} e_{i}\right\| .
$$

Suppose furthermore that for any $x=\sum a_{i} e_{i}$ the series actually converges unconditionally, i.e., for any permutation $\sigma$ of $\mathbb{N}$ the series $\sum a_{\sigma(i)} e_{\sigma(i)}$ converges to $x$. Then the basic sequence is said to be unconditional.

Again, being an unconditional basis for some closed subspace (which will be denoted by "unconditional basic sequence") is equivalent to there being a constant $K \geq 1$ such that for all $n, A \subseteq\{0, \ldots, n\}$ and $a_{0}, \ldots, a_{n} \in \mathbb{R}$,

$$
\left\|\sum_{i \in A} a_{i} e_{i}\right\| \leq K\left\|\sum_{i=0}^{n} a_{i} e_{i}\right\| .
$$

We will in general only work with normalised basic sequences, i.e., $\left\|e_{i}\right\| \equiv 1$, which can always be obtained by taking $e_{i}^{\prime}:=e_{i} /\left\|e_{i}\right\|$.

Given some vector $x \in \operatorname{span}\left(e_{i}\right)$ let its support, $\operatorname{supp}(x)$, be the set of indices $i$ with $e_{i}^{*}(x) \neq 0$. For $k \in \mathbb{N}$ and $x, y \in \operatorname{span}\left(e_{i}\right)$ we write $k<x$ if $k<\min \operatorname{supp}(x)$, and $x<y$ if $\max \operatorname{supp}(x)<\min \operatorname{supp}(y)$. A block basis, $\left(x_{i}\right)$, over a basis $\left(e_{i}\right)$ is a finite or infinite sequence of vectors in $\operatorname{span}\left(e_{i}\right)$ with $x_{0}<x_{1}<x_{2}<\cdots$. This sequence will also be basic and in fact unconditional in case $\left(e_{i}\right)$ is so.

Two basic sequences $\left(e_{i}\right)$ and $\left(t_{i}\right)$ are called equivalent, in symbols $\left(e_{i}\right) \approx\left(t_{i}\right)$, provided a series $\sum a_{i} e_{i}$ converges if and only if $\sum a_{i} t_{i}$ converges. This can also be stated as saying that $T: e_{i} \mapsto t_{i}$ extends to an invertible linear operator between $\left[e_{i}\right]$ and $\left[t_{i}\right]$. The quantity $\|T\| \cdot\left\|T^{-1}\right\|$ is then the constant of equivalence between the two bases.

An unconditional basis that is equivalent to all of its subsequences is said to be subsymmetric. A simple diagonalisation argument then shows that it must be uniformly equivalent to all of its subsequences.

Two basic sequences $\left(e_{i}\right)$ and $\left(t_{i}\right)$ are said to be permutatively equivalent if there is some permutation $\sigma$ of $\mathbb{N}$ such that $\left(e_{i}\right)$ and $\left(t_{\sigma(i)}\right)$ are equivalent.

2. Incomparable and minimal subspaces. Two Banach spaces $X$ and $Y$ are called incomparable in case neither of them embed isomorphically into the other. $X$ is said to be minimal if it embeds into all of its infinitedimensional subspaces and $X$ itself is infinite-dimensional.

Our proof of the first theorem will proceed by a reduction to an analysis of Borel partial orders due to L. Harrington, D. Marker and S. Shelah [14]. Instrumental in our reduction will be the determinacy result of Gowers [10] on certain games in Banach spaces, which will guarantee that some choices 
can be done uniformly, a fact that is needed for definability purposes. Moreover, we will use some ideas of J. López-Abad [19] on coding reals with inevitable subsets of the unit sphere of a Banach space, which in turn relies on the results of Odell and Schlumprecht [20].

A nice presentation of the progress made in the geometric theory of Banach spaces in the nineties can be found in the article [11] by W. T. Gowers.

We mention that it was shown by a simpler argument in [9] by V. Ferenczi and the author that any Banach space either contains a minimal subspace or a continuum of non-isomorphic subspaces.

To simplify notation, we write $X \sqsubseteq Y$ if $X$ embeds isomorphically into $Y$, and we always suppose the spaces we are working with to be separable infinite-dimensional. Then $\sqsubseteq$ restricted to the standard Borel space of subspaces of some separable Banach space becomes an analytic quasi-order, i.e., transitive and reflexive. So Theorem 1 amounts to saying that $\sqsubseteq$ has either a minimal element or a perfect antichain.

Suppose $\left(e_{i}\right)$ is a normalised basic sequence with norm denoted by $\|\cdot\|$. We call a normalised block vector $x$ with finite support rational if it is a scalar multiple of a finite linear combination of $\left(e_{i}\right)$ with rational coordinates. Notice that there are only countably many rational (finite) block vectors, which we can gather in a set $Q$ and give it the discrete topology. Let $\mathrm{bb}_{\mathbb{Q}}\left(e_{i}\right)$ be the set of block bases of $\left(e_{i}\right)$ consisting of rational normalised block vectors. This is easily seen to be a closed subspace of $Q^{\mathbb{N}}$, which is itself a Polish space. Moreover, the canonical function sending $X \in \mathrm{bb}_{\mathbb{Q}}\left(e_{i}\right)$ to its closed span in $\mathfrak{B}$ is Borel, so the relations of isomorphism, etc., become analytic on $\mathrm{bb}_{\mathbb{Q}}\left(e_{i}\right)$.

We recall the following classical facts: Any infinite-dimensional Banach space contains an infinite normalised basic sequence $\left(e_{i}\right)$. Moreover, if $Y$ is any subspace of $\left[e_{i}\right]$, then it contains an isomorphic perturbation of a block basic sequence of $\left(e_{i}\right)$. Again, any block basic sequence is equivalent to some member of $\operatorname{bb}_{\mathbb{Q}}\left(e_{i}\right)$. So this explains why we can concentrate on $\operatorname{bb}_{\mathbb{Q}}\left(e_{i}\right)$ if we are only looking for minimal subspaces.

For $X, Y \in \operatorname{bb}_{\mathbb{Q}}\left(e_{i}\right)$, let $X \leq Y$ if $X$ is a blocking of $Y$, i.e., if any element of $X$ is a linear combination over $Y$. Note that this does not imply that they are rational block vectors over $Y$, but only over $\left(e_{i}\right)$. Moreover, if $Y=\left(y_{i}\right), X=\left(x_{i}\right) \in \operatorname{bb}_{\mathbb{Q}}\left(e_{i}\right)$, set $Y \leq^{*} X$ if $\left(y_{i}\right)_{i \geq k} \leq X$ for some $k$. Also, for $\Delta=\left(\delta_{i}\right)$ an infinite sequence of strictly positive reals write $d(X, Y)<\Delta$ if $\left\|x_{i}-y_{i}\right\|<\delta_{i}$ for all $i$.

Write $X \approx Y$ if the bases are equivalent and $X \cong Y$ if they span isomorphic spaces. Then a classical perturbation argument shows that there is some $\Delta$, depending only on the constant of the basis, such that for any $X, Y \in \operatorname{bb}_{\mathbb{Q}}\left(e_{i}\right)$ if $d(X, Y)<\Delta$, then $X \approx Y$ and in particular $X \cong Y$. Write 
also $X=\left(x_{i}\right) \simeq Y=\left(y_{i}\right)$ if $\exists k \forall i \geq k x_{i}=y_{i}$. Then, evidently, $X \simeq Y$ implies $X \approx Y$.

For a subset $A \subseteq \operatorname{bb}_{\mathbb{Q}}\left(e_{i}\right)$ let

$$
\begin{aligned}
A^{*} & =\left\{Y \in \operatorname{bb}_{\mathbb{Q}}\left(e_{i}\right) \mid \exists X \in A X \cong Y\right\}, \\
A_{\Delta} & =\left\{Y \in \operatorname{bb}_{\mathbb{Q}}\left(e_{i}\right) \mid \exists X \in A d(X, Y)<\Delta\right\} .
\end{aligned}
$$

Notice that if $A$ is analytic then so are both $A^{*}$ and $A_{\Delta}$. Again $[Y]=\{X \in$ $\left.\operatorname{bb}_{\mathbb{Q}}\left(e_{i}\right) \mid X \leq Y\right\}$. Such an $A$ is said to be large in $[Y]$ if for any $X \in[Y]$ we have $[X] \cap A \neq \emptyset$.

For $\left(e_{i}\right)$ a given normalised basis, $A \subseteq \operatorname{bb}_{\mathbb{Q}}\left(e_{i}\right)$ and $X \in \operatorname{bb}_{\mathbb{Q}}\left(e_{i}\right)$, the Gowers game $\partial_{X}^{A}$ is defined as follows: in the $k$ th move of the game, player I plays a rational normalised block vector $y_{k}$ of $\left(e_{i}\right)$ such that $y_{k-1}<y_{k}$ and $y_{k}$ is a block on $X$. Player II responds by either doing nothing or playing a rational normalised block vector $x$ such that $x \in\left[y_{l+1}, \ldots, y_{k}\right]$ where $l$ was the last move where II played a vector. So player II wins the game if in the end she has produced an infinite rational block basis $X=\left(x_{i}\right) \in A$. This is an equivalent formulation due to J. Bagaria and J. López-Abad [1] of Gowers's original game.

Gowers [10] proved that if $A \subseteq \operatorname{bb}_{\mathbb{Q}}\left(e_{i}\right)$ is analytic, large in $[Y]$, and $\Delta$ is given, then for some $X \in[Y]$, II has a winning strategy in the game $\partial_{X}^{A_{\Delta}}$.

We also mention a result of Odell and Schlumprecht [20,21] obtained from their solution to the distortion problem: If $E$ is an infinite-dimensional Banach space not containing $c_{0}$, there are an infinite-dimensional subspace $F$ and $A, B \subseteq \mathcal{S}_{F}$ of positive distance such that any infinite-dimensional subspace of $F$ intersects both $A$ and $B$.

The following was shown in [9]:

Lemma 5. (MA) Let $A \subseteq \mathrm{bb}_{\mathbb{Q}}\left(e_{i}\right)$ be linearly ordered under $\leq^{*}$ of cardinality strictly less than the continuum. Then there is some $X \in \mathrm{bb}_{\mathbb{Q}}\left(e_{i}\right)$ such that $X \leq{ }^{*} Y$ for all $Y \in A$.

From this lemma one gets the following:

Lemma 6. ( $\mathrm{MA}+\neg \mathrm{CH})$ Suppose $\mathbb{W} \subseteq \mathrm{bb}_{\mathbb{Q}}\left(e_{i}\right)$ is a $\Sigma_{2}^{1}$ set large in some $[Y]$, and $\Delta>0$. Then II has a winning strategy in $\partial_{X}^{\mathbb{W}_{\Delta}^{*}}$ for some $X \in[Y]$.

Proof. Let $\mathbb{W}=\bigcup_{\omega_{1}} \mathbb{V}_{\xi}$ be a decomposition of $\mathbb{W}$ as an increasing union of $\aleph_{1}$ Borel sets. We claim that some $\mathbb{V}_{\xi}^{*}$ is large in $[Z]$ for some $Z \in[Y]$, which by Gowers's theorem will be enough to prove the lemma. So suppose not and find $Y_{0} \in[Y]$ such that $\left[Y_{0}\right] \cap \mathbb{V}_{0}^{*}=\emptyset$. Repeating the same process and diagonalising at limits, we find $Y_{\xi} \in[Y]$ for $\xi<\omega_{1}$ such that $\left[Y_{\xi}\right] \cap \mathbb{V}_{\xi}^{*}=\emptyset$ 
and $Y_{\xi} \leq^{*} Y_{\eta}$ for $\eta<\xi$. By the above lemma there is some $Y_{\infty}=\left(y_{i}\right) \in[Y]$ with $Y_{\infty} \leq{ }^{*} Y_{\xi}$ for all $\xi<\omega_{1}$.

We claim that $\left[\left(y_{2 i}\right)\right] \cap \mathbb{W}=\emptyset$. Otherwise, for $Z=\left(z_{i}\right) \in\left[\left(y_{2 i}\right)\right] \cap \mathbb{W}$ find $\xi<\omega_{1}$ such that $Z \in \mathbb{V}_{\xi}$. Now as $\left(y_{i}\right) \leq^{*} Y_{\xi}$ there is some $k$ with $\left(y_{i}\right)_{i \geq k} \leq Y_{\xi}$, whence

$$
\left(y_{2 i}\right) \simeq\left(y_{k}, y_{k+1}, y_{k+2}, \ldots, y_{2 k-1}, y_{2 k}, y_{2(k+1)}, y_{2(k+2)}, \ldots\right) \leq Y_{\xi} .
$$

One now easily sees that there is some $\left(x_{i}\right)$ with

$$
\left(z_{i}\right) \simeq\left(x_{i}\right) \leq\left(y_{k}, y_{k+1}, y_{k+2}, \ldots, y_{2 k-1}, y_{2 k}, y_{2(k+1)}, y_{2(k+2)}, \ldots\right),
$$

whereby $\left(x_{i}\right) \in \mathbb{V}_{\xi}^{*}$, contradicting $\mathbb{V}_{\xi}^{*} \cap\left[Y_{\xi}\right]=\emptyset$.

Therefore $\left[\left(y_{2 i}\right)\right] \cap \mathbb{W}=\emptyset$, again contradicting the largeness of $\mathbb{W}$.

Lemma 7. (MA $+\neg \mathrm{CH})$ Suppose that $\left(e_{i}\right)$ is a basic sequence such that $\left[e_{i}\right]$ does not contain a minimal subspace. Then for any $W \in \mathrm{bb}_{\mathbb{Q}}\left(e_{i}\right)$ there are a $Y \in[W]$ and a Borel function $g:[Y] \rightarrow[Y]$ such that for all $Z \in[Y]$,

$$
g(Z) \leq Z \& Z \nsubseteq g(Z) .
$$

Proof. As $c_{0}$ is minimal, $\left[e_{i}\right]$ does not contain $c_{0}$. Therefore, by the solution to the distortion problem by Odell and Schlumprecht [20], we can, by replacing $\left(e_{i}\right)$ by a block, suppose that we have two positively separated sets $F_{0}, F_{1}$ of the unit sphere such that for any $X \in \mathrm{bb}_{\mathbb{Q}}\left(e_{i}\right)$ there are rational normalised blocks $x, y$ on $X$ with $x \in F_{0}$ and $y \in F_{1}$. We call such sets inevitable.

Let now

$$
\mathbb{D}=\left\{X=\left(x_{i}\right) \in \operatorname{bb}_{\mathbb{Q}}\left(e_{i}\right) \mid \forall i x_{i} \in F_{0} \cup F_{1}\right\}
$$

and for $X \in \mathbb{D}$ let $\alpha(X) \in 2^{\mathbb{N}}$ be defined by

$$
\alpha(X)(i)=0 \Leftrightarrow x_{i} \in F_{0} .
$$

Then $\mathbb{D}$ is easily seen to be a closed subset of $\operatorname{bb}_{\mathbb{Q}}\left(e_{i}\right)$ and $\alpha: \mathbb{D} \rightarrow 2^{\mathbb{N}}$ to be continuous. Furthermore, by the inevitability of $F_{0}$ and $F_{1}$, we see that $\mathbb{D}$ is large in every $[Y]$.

Let $\mathbb{Q}_{*}^{<\mathbb{N}}$ be the set of finite non-identically zero sequences of rational numbers, endowed with the discrete topology. Then $\left(\mathbb{Q}_{*}^{<\mathbb{N}}\right)^{\mathbb{N}}$ is Polish. For any $Y \in \operatorname{bb}_{\mathbb{Q}}\left(e_{i}\right)$ and $\left(\underline{\lambda}_{i}\right) \in\left(\mathbb{Q}_{*}^{<\mathbb{N}}\right)^{\mathbb{N}}$ define the block basis $\left(\underline{\lambda}_{i}\right) \cdot Y$ of $Y$ in the obvious way, by taking the successive linear combinations given by $\left(\underline{\lambda}_{i}\right)$.

Fix also some perfect set $P$ of almost disjoint subsets of $\mathbb{N}$ seen as a subset of $2^{\mathbb{N}}$ and let $\beta: P \leftrightarrow\left(\mathbb{Q}_{*}^{<\mathbb{N}}\right)^{\mathbb{N}}$ be a Borel isomorphism. Again

$$
\mathbb{E}=\{X \in \mathbb{D} \mid \alpha(X) \in P\}
$$

is closed and large in $\mathrm{bb}_{\mathbb{Q}}\left(e_{i}\right)$. Moreover, the set

$$
\mathbb{W}=\left\{X=\left(x_{i}\right) \in \operatorname{bb}_{\mathbb{Q}}\left(e_{i}\right) \mid\left(x_{2 i}\right) \in \mathbb{E} \wedge\left(x_{2 i+1}\right) \not \beta \circ \alpha\left(\left(x_{2 i}\right)\right) \cdot\left(x_{2 i+1}\right)\right\}
$$

is coanalytic. We claim that it is large in $\operatorname{bb}_{\mathbb{Q}}\left(e_{i}\right)$. 
To see this, let $Y \in \mathrm{bb}_{\mathbb{Q}}\left(e_{i}\right)$ be given and take, by inevitability of $F_{0}$ and $F_{1}$, some $\left(z_{i}\right) \in[Y]$ with $z_{3 i} \in F_{0}$ and $z_{3 i+1} \in F_{1}$. As $\left[z_{3 i+2}\right]$ is not minimal, there is some $X \leq\left(z_{3 i+2}\right)$ such that $\left(z_{3 i+2}\right) \nsubseteq X$. Take some $\left(\underline{\lambda}_{i}\right) \in\left(\mathbb{Q}_{*}^{<\mathbb{N}}\right)^{\mathbb{N}}$ such that

$$
\left(\underline{\lambda}_{i}\right) \cdot\left(z_{3 i+2}\right) \approx X
$$

whence

$$
\left(z_{3 i+2}\right) \nsubseteq\left(\underline{\lambda}_{i}\right) \cdot\left(z_{3 i+2}\right) .
$$

We can now define some $\left(v_{i}\right)$ by letting

$$
v_{2 i+1}=z_{3 i+2}
$$

and either $v_{2 i}=z_{3 i}$ or $v_{2 i}=z_{3 i+1}$ in such a way that

$$
\beta \circ \alpha\left(\left(v_{2 i}\right)\right)=\left(\underline{\lambda}_{i}\right) \text {. }
$$

This ensures that $\left(v_{i}\right) \in \mathbb{W}$. So as $\left(v_{i}\right) \leq\left(z_{i}\right)$, it is in $[Y]$ and $\mathbb{W}$ is indeed large.

Take now some $\Delta=\left(\delta_{i}\right)$ depending on the basis constant such that $d\left(Y, Y^{\prime}\right)<\Delta$ implies $Y \approx Y^{\prime}$, and moreover $\delta_{i}<\frac{1}{2} d\left(F_{0}, F_{1}\right)$. By Lemma 6 we can find a $Y \in \operatorname{bb}_{\mathbb{Q}}\left(e_{i}\right)$ such that II has a winning strategy $\sigma$ in the game $\partial_{Y}^{\mathbb{W}^{*}}$.

Suppose that $X=\left(x_{i}\right)$ has been played by II according to the strategy $\sigma$ as a response to $Z$ played by I. As $\sigma$ is winning for II, $X \in \mathbb{W}_{\Delta}^{*}$. Define $\gamma(X) \in 2^{\mathbb{N}}$ by $\gamma(X)(i)=0$ if $d\left(x_{2 i}, F_{0}\right)<\delta_{2 i}$ and $\gamma(X)(i)=1$ otherwise. Then $\gamma$ is Borel from $\mathbb{W}_{\Delta}^{*}$ to $2^{\mathbb{N}}$, and furthermore, since $P$ was chosen to consist of almost disjoint subsets of $\mathbb{N}$, there is a unique $\gamma^{*}(X) \in P$ such that

$$
\exists k \forall i \geq k \gamma(X)(i)=\gamma^{*}(X)(i) .
$$

Again $X \mapsto \gamma^{*}(X)$ is Borel.

Take some $U=\left(u_{i}\right) \in \mathbb{W}$ such that $\exists m \forall n \geq m\left\|u_{n}-x_{n}\right\|<\delta_{n}$. Then

$$
\alpha\left(\left(u_{2 i}\right)\right)=\gamma^{*}(X), \quad\left(u_{2 i+1}\right) \approx\left(x_{2 i+1}\right), \quad\left(u_{2 i+1}\right) \nsubseteq \beta \circ \alpha\left(\left(u_{2 i}\right)\right) \cdot\left(u_{2 i+1}\right) .
$$

So since the basis is invariant up to equivalence by $\Delta$-perturbations we have

$$
\left(x_{2 i+1}\right) \nsubseteq \beta \circ \gamma^{*}(X) \cdot\left(x_{2 i+1}\right) .
$$

Let $V \in[X]$ be the normalisation of $\beta \circ \gamma^{*}(X) \cdot\left(x_{2 i+1}\right)$. The function $g: Z \mapsto$ $V$ is Borel and obviously

$$
V \approx \beta \circ \gamma^{*}(X) \cdot\left(x_{2 i+1}\right) \leq\left(x_{2 i+1}\right) \leq Z,
$$

and as

$$
\left(x_{2 i+1}\right) \nsubseteq \beta \circ \gamma^{*}(X) \cdot\left(x_{2 i+1}\right),
$$

also $Z \nsubseteq V$.

A Banach space is called quasi-minimal if any two subspaces have further isomorphic subspaces. The following is a standard observation. 
Lemma 8. Suppose $\left[e_{i}\right]$ is quasi-minimal. Then $\sqsubseteq$ is downwards $\sigma$-directed on $\operatorname{bb}_{\mathbb{Q}}\left(e_{i}\right)$, i.e., any countable family has a common minorant.

Proof. Suppose that $Y_{i} \in \mathrm{bb}_{\mathbb{Q}}\left(e_{i}\right)$ are given, and define inductively $Z_{i} \in$ $\left[Y_{0}\right]$ such that $Z_{i} \sqsubseteq Y_{i}$ and $Z_{i+1} \leq Z_{i}$. Take some $Z=\left(z_{i}\right) \leq{ }^{*} Z_{n}$ for all $n$ and notice as in the proof of Lemma 6 that $\left(z_{2 i}\right) \sqsubseteq Z_{n}$ for all $n$.

Lemma 9. If $R$ is a downwards $\sigma$-directed Borel quasi-order on a standard Borel space $X$, then $R$ has either a perfect antichain or a minimal element.

Proof. This is a simple consequence of the results of L. Harrington, D. Marker and S. Shelah [14], as we will see. Suppose that $R$ did not have a perfect antichain. Then by their results there is a countable partition $X=\bigcup X_{n}$ into Borel sets so that $R$ is total on each piece, i.e., $R$ can be written as a countable union of $R$-chains.

By another of their results this implies that for some countable ordinal $\alpha$ there are Borel functions $f_{n}: X_{n} \rightarrow 2^{\alpha}$ such that for any $x, y \in X_{n}$,

$$
y R x \Leftrightarrow x \leq_{\text {lex }} y,
$$

where $\leq_{\text {lex }}$ is the usual lexicographical ordering. In their terminology, $R$ is linearisable on each $X_{n}$.

One can easily check that any subset of $2^{\alpha}$ has a countable subset cofinal with respect to $\leq_{\text {lex }}$, so pulling it back by $f_{n}$ it becomes coinitial in $R \uparrow_{X_{n}}$. Putting all these sets together one gets a countable subset of $X$ coinitial with respect to $R$. So by downwards $\sigma$-directedness there is therefore a minimal element in $X$.

In this connection, we should also mention an unpublished result of Khalid Kada (from his doctoral thesis at the University of Paris 6), which is the Borel version of Dilworth's theorem: If in a Borel ordering $R$ all antichains are of size at most $n$ (for some finite $n$ ), then there is a decomposition of the underlying space into $n$ Borel $R$-chains.

After this series of lemmas we can now prove the theorem:

Theorem 10. Let $X$ be an infinite-dimensional Banach space. Then $X$ contains either a minimal subspace or a continuum of pairwise incomparable subspaces.

Proof. By Gowers's quadrichotomy, $X$ contains either a quasi-minimal subspace or a subspace with a basis such that any two disjointly supported subspaces are totally incomparable (see Gowers [10, Theorem 7.2] and the fact that H.I. spaces are quasi-minimal). In the latter case, any perfect set of almost disjoint subsets of $\mathbb{N}$ will give rise to subsequences of the basis spanning totally incomparable spaces, which would prove the theorem. So we can suppose that $X=\left[e_{i}\right]$ is quasi-minimal for some basis $\left(e_{i}\right)$. If $X$ 
does not contain a minimal subspace, we can choose $Z \in \mathrm{bb}_{\mathbb{Q}}\left(e_{i}\right)$ and a Borel function $g$ as in Lemma 7 (under MA $+\neg \mathrm{CH}$ of course). So define the following property on subsets $\boldsymbol{A}, \boldsymbol{B}$ of $[Z]^{2}$ :

$$
\begin{aligned}
& \Phi(\boldsymbol{A}, \boldsymbol{B}) \Leftrightarrow \\
& \forall Y, V, W \in[Z][Y \boldsymbol{A} V \& V \boldsymbol{A} W \rightarrow \neg Y \boldsymbol{B} W] \& \forall Y \in[Z](\neg Y \boldsymbol{A} g(Y)) .
\end{aligned}
$$

We now wish to apply the second reflection theorem (see Kechris [17, Theorem (35.16) and the comments that follow]). For this we need to verify that $\Phi$ is

(i) hereditary, i.e., if $\Phi(\boldsymbol{A}, \boldsymbol{B})$ and $\boldsymbol{A}^{\prime} \subseteq \boldsymbol{A}, \boldsymbol{B}^{\prime} \subseteq \boldsymbol{B}$, then also $\Phi\left(\boldsymbol{A}^{\prime}, \boldsymbol{B}^{\prime}\right)$;

(ii) upward continuous in the second variable, i.e., if $\Phi\left(\boldsymbol{A}, \boldsymbol{B}_{n}\right) \& \boldsymbol{B}_{n}$ $\subseteq \boldsymbol{B}_{n+1}$ then also $\Phi\left(\boldsymbol{A}, \bigcup \boldsymbol{B}_{n}\right)$;

(iii) $\boldsymbol{\Pi}_{1}^{1}$ on $\boldsymbol{\Sigma}_{1}^{1}$, i.e., if $\boldsymbol{A}, \boldsymbol{B} \subseteq \mathbb{N}^{\mathbb{N}} \times[Z]^{2}$ are $\boldsymbol{\Sigma}_{1}^{1}$, then the set

$$
\mathcal{A}_{\Phi}=\left\{(x, y) \in \mathbb{N}^{\mathbb{N}} \times \mathbb{N}^{\mathbb{N}} \mid \Phi\left(\boldsymbol{A}_{x}, \boldsymbol{B}_{y}\right)\right\}
$$

is $\Pi_{1}^{1}$.

Conditions (i) and (ii) should be clear. Condition (iii) is also easily seen, by noting that

$$
\begin{aligned}
(x, y) & \in \mathcal{A}_{\Phi} \Leftrightarrow \\
& \forall Y, V, W \in[Z][(x, Y, V) \notin \boldsymbol{A} \vee(x, V, W) \notin \boldsymbol{A} \vee(y, Y, W) \notin \boldsymbol{B}] \\
& \& \forall Y \in[Z](x, Y, g(Y)) \notin \boldsymbol{A} .
\end{aligned}
$$

Now, since $\Phi(\sqsubseteq, \nsubseteq)$, there is by reflection some Borel set $R$ containing $\sqsubseteq$ such that $\Phi(R, \complement R)$. But then $R$ is a Borel quasi-order, downwards $\sigma$-directed, as it contains $\sqsubseteq$, and without a minimal element, as witnessed by $g$. So $R$ has a perfect antichain by the previous lemma, which then is an antichain for $\sqsubseteq$ as well.

The statement is therefore proved under the additional hypothesis of Martin's axiom and the negation of the continuum hypothesis. We will see that this is in fact sufficient to prove the theorem. By standard metamathematical facts and Shoenfield's absoluteness theorem it is enough to show that the statement we wish to prove is $\Sigma_{2}^{1}$.

It was proved by Ferenczi and the author in [9] that the property of having a block minimal subspace is $\Sigma_{2}^{1}$. To prove this, one uses Gowers's determinacy result and codings as above in order to continuously find an isomorphism between the space and a certain subspace, hence witnessing the minimality. This proof can be trivially modified to show that the property of having a minimal (i.e., not necessarily block minimal) subspace is also $\Sigma_{2}^{1}$. For now we only have to choose not a code for a subspace and an isomorphism, but a code for a subspace and an embedding. For the convenience of the reader, we have included the proof of this in an appendix. 
On the other hand, the property of having a perfect antichain is obviously $\Sigma_{2}^{1}$ by just counting quantifiers. So these remarks finish the proof.

V. Ferenczi has proved in a recent preprint [6] that for any infinite-dimensional Banach space, either the space contains a minimal subspace, or $E_{0}$ Borel reduces to the relation of isomorphism between its subspaces. This, along with Theorem 1, reinforces our belief in the following conjecture:

Conjecture 11. $E_{0}$ Borel reduces to the relation of isomorphism between the subspaces of any non-Hilbertian space.

A proof of this would certainly be a substantial improvement to the solution of the homogeneous space problem.

3. Ramsey type results. We will show two Ramsey type results and afterwards some applications to Banach space theory.

It is well known that there are no nice Ramsey properties for the product space $[\mathbb{N}]^{\mathbb{N}} \times[\mathbb{N}]^{\mathbb{N}}$ as opposed to the simple Ramsey space $[\mathbb{N}]^{\mathbb{N}}$. That is, there are even quite simple relations not admitting a square $[A]^{\mathbb{N}} \times[B]^{\mathbb{N}}$ that is either included in or disjoint from the relation.

We are interested in the case when the relation on the product is in fact a definable equivalence relation. Here the right question seems to be when there is a cube $[A]^{\mathbb{N}}$ contained in one equivalence class. Now, if one lets two subsets of $\mathbb{N}$ be equivalent if and only if they have the same minimal element, then the relation has exactly $\aleph_{0}$ classes and does not admit a homogeneous set.

On the other hand, if the relation is invariant under finite changes, such as $E_{0}$, then there are bigger chances that it should have a homogeneous set. We will show that in the case of analytic equivalence relations, $E_{0}$ is in fact the minimal counterexample to the Ramsey property, in the sense that, if an analytic equivalence relation is invariant under finite changes and does not admit a homogeneous set, then $E_{0}$ Borel reduces to it. In the same vein, it is shown that if an analytic equivalence relation does not admit a cube on which it has only countably many classes, then it has at least a perfect set of classes. We notice that both of these results are relatively direct consequences of the Silver and Glimm-Effros dichotomies in the case of the equivalence relation being Borel. But our results are motivated by applications to isomorphism of separable Banach spaces, which is true analytic, and the dichotomies are known not to hold in this generality.

The following result grew out of a discussion with S. Todorcevic, who also independently found a proof [22].

THEOREM 12. Let $\boldsymbol{E}$ be an analytic equivalence relation on $[\mathbb{N}]^{\mathbb{N}}$. Then either $\boldsymbol{E}$ has a continuum of classes or there is some $A \in[\mathbb{N}]^{\mathbb{N}}$ such that $\boldsymbol{E}$ 
only has a countable number of classes on $[A]^{\mathbb{N}}$. Moreover, the $E_{0}$ class of $A$ will intersect every $\boldsymbol{E}$-class of $[A]^{\mathbb{N}}$.

Proof. We will first prove the theorem under MA $+\neg \mathrm{CH}$. Assume that $\boldsymbol{E}$ does not have a continuum of classes. By Burgess's theorem (Exercise $(35.21)$ in [17]) we can suppose that $\boldsymbol{E}$ has at most $\aleph_{1}$ classes, $\left(\mathbb{C}_{\xi}\right)_{\omega_{1}}$. Define $P_{\xi}(A) \leftrightarrow[A]_{E_{0}} \cap \mathbb{C}_{\xi} \neq \emptyset$ and notice that this is an analytic $E_{0}$-invariant property. We can by simple diagonalisation find $\left(A_{\xi}\right)_{\omega_{1}}$ with $A_{\xi} \subseteq^{*} A_{\eta}$ for $\eta<\xi<\omega_{1}$ such that for all $\xi<\omega_{1}$ either $\left[A_{\xi}\right]^{\mathbb{N}} \subseteq P_{\xi}$ or $\left[A_{\xi}\right]^{\mathbb{N}} \subseteq \complement P_{\xi}$. And by MA $+\neg \mathrm{CH}$ there is an $A \subseteq^{*} A_{\xi}$ for all $\xi<\omega_{1}$.

Notice that by $E_{0}$-invariance of $P_{\xi}$, if $B \subseteq^{*} A$ and $[A]^{\mathbb{N}} \subseteq P_{\xi}$ or $[A]^{\mathbb{N}} \subseteq \complement P_{\xi}$, then also $[B]^{\mathbb{N}} \subseteq P_{\xi}$, respectively $[B]^{\mathbb{N}} \subseteq \complement P_{\xi}$. Therefore for all $\xi<\omega_{1}$ either $[A]^{\mathbb{N}} \subseteq P_{\xi}$ or $[A]^{\mathbb{N}} \subseteq \complement P_{\xi}$.

Suppose now that $B \in[A]^{\mathbb{N}}, B \in \mathbb{C}_{\xi}$. Then $P_{\xi}(B)$ and therefore $[A]^{\mathbb{N}} \subseteq P_{\xi}$ and $P_{\xi}(A)$, i.e., $\exists A^{\prime}\left(A^{\prime} E_{0} A \& A^{\prime} \boldsymbol{E} B\right)$. This shows that $[A]_{E_{0}}$ will intersect every $\boldsymbol{E}$-class of $[A]^{\mathbb{N}}$. Moreover, as the properties $P_{\xi}$ are stabilised on $[A]^{\mathbb{N}}$, for any $B \in[A]^{\mathbb{N}}$ we have $\left[[A]_{E_{0}}\right]_{\boldsymbol{E}}=\left[[B]_{E_{0}}\right]_{\boldsymbol{E}}$. Since $[A]_{E_{0}}$ is countable, this also implies that there are only countably many $\boldsymbol{E}$-classes of $[A]^{\mathbb{N}}$.

Let us now check that the statement of the theorem is absolute. Saying that $\boldsymbol{E}$ has a continuum of classes is equivalent to saying that there is a compact perfect set $K \subseteq[\mathbb{N}]^{\mathbb{N}}$ consisting of pairwise $\boldsymbol{E}$-inequivalent points:

$$
\exists K \subseteq[\mathbb{N}]^{\mathbb{N}} \text { compact, perfect } \forall C, D \in K(C=D \vee \neg C \boldsymbol{E} D) .
$$

This is obviously a $\Sigma_{2}^{1}$ statement.

For the other case, we wish to express the existence of some $A$ such that $[A]_{E_{0}}$ intersects every $\boldsymbol{E}$-class of $[A]^{\mathbb{N}}$. However, on the face of it this is only a $\Sigma_{3}^{1}$ statement, so we need to work a bit to reduce the complexity.

Choose first some Borel set $\mathcal{B} \subseteq[\mathbb{N}]^{\mathbb{N}} \times[\mathbb{N}]^{\mathbb{N}} \times \mathbb{N}^{\mathbb{N}}$ such that for $C, D \in[\mathbb{N}]^{\mathbb{N}}$ we have $C \boldsymbol{E} D \Leftrightarrow \exists \alpha \in \mathbb{N}^{\mathbb{N}} \mathcal{B}(C, D, \alpha)$. Notice that as $[A]_{E_{0}}$ intersects every $\boldsymbol{E}$-class of $[A]^{\mathbb{N}}$, by the Jankov-von Neumann selection theorem there is a $C$-measurable selector $f:[A]^{\mathbb{N}} \rightarrow \mathbb{N}^{\mathbb{N}}$ such that for any $D \in[A]^{\mathbb{N}}$ there is an $A^{\prime} E_{0} A$ with $\mathcal{B}\left(D, A^{\prime}, f(D)\right)$. That is, we can choose a witness to $D$ being $\boldsymbol{E}$-equivalent to some $A^{\prime} E_{0} A$ in a $C$-measurable way. But any $C$-measurable function can, by the theorem of Ellentuck and Louveau, be rendered continuous on a cube, i.e., there is some $B \in[A]^{\mathbb{N}}$ such that the restriction of $f$ to $[B]^{\mathbb{N}}$ is continuous. Moreover, by the above, $\left[[A]_{E_{0}}\right]_{\boldsymbol{E}}=\left[[B]_{E_{0}}\right]_{\boldsymbol{E}}$, whence the other possibility can be written as

$\exists A, B \in[\mathbb{N}]^{\mathbb{N}} \exists f:[B]^{\mathbb{N}} \rightarrow \mathbb{N}^{\mathbb{N}}$ continuous

$$
\left(B \subseteq A \&\left[[A]_{E_{0}}\right]_{\boldsymbol{E}}=\left[[B]_{E_{0}}\right]_{\boldsymbol{E}} \& \forall D \in[B]^{\mathbb{N}} \exists A^{\prime} \in[A]_{E_{0}} \mathcal{B}\left(D, A^{\prime}, f(D)\right)\right) .
$$

Since $E_{0}$-classes are countable and can be effectively enumerated given any element of the class, the statement is $\Sigma_{2}^{1}$. Therefore, by Shoenfield's abso- 
luteness theorem and standard metamathematical facts it is enough to prove the result under $\mathrm{MA}+\neg \mathrm{CH}$.

Our next results render explicit the connection with the Borel reducibility ordering.

Definition 13. For $A, B \subseteq \mathbb{N}$ set

$$
A E_{0}^{\prime} B \Leftrightarrow \exists n|A \cap n|=|B \cap n| \wedge A \backslash n=B \backslash n .
$$

It is easy to see that the $E_{0}^{\prime}$-class of any infinite-coinfinite subset of $\mathbb{N}$ is dense in $[\mathbb{N}]^{\mathbb{N}}$ and in fact $E_{0}^{\prime}$ is generically ergodic. Moreover, $E_{0}^{\prime}$ is just a refinement of $E_{0}$.

LEMMA 14. $E_{0}^{\prime}$ is generically ergodic (i.e., any invariant set with the Baire property is either meagre or comeagre) and all classes $[A]_{E_{0}^{\prime}}$, for $A$ infinite-coinfinite, are dense.

Proof. Since $[\mathbb{N}]^{\mathbb{N}}$ is cocountable in $2^{\mathbb{N}}$ we can restrict our attention to it. Suppose that some invariant set $\mathcal{A}$ is non-meagre; then there is some $a \subseteq[0, n]$ such that $\mathcal{A}$ is comeagre in $D_{a, n}=\left\{A \in[\mathbb{N}]^{\mathbb{N}} \mid A \cap[0, n]=a\right\}$. So for any $D_{b, m}$ there are $c, d \subseteq[0, k], \max (n, m)<k$, such that $a \subseteq c$, $b \subseteq d,|c|=|d|$. Now for any $A \in[\{k+1, k+2, \ldots\}]^{\mathbb{N}}$ we have $\phi(c \cup A):=$ $(d \cup A) E_{0}^{\prime}(c \cup A)$ and $\phi$ is a homeomorphism of $D_{c, k} \subseteq D_{a, n}$ with $D_{d, k}$. But that means that the image of $\mathcal{A}$ is comeagre in $D_{d, k} \subseteq D_{b, m}$ and is included in the saturation of $\mathcal{A}$, which is $\mathcal{A}$. So $\mathcal{A}$ is comeagre in the space.

If $A$ is infinite-coinfinite, then for any $D_{a, n}$ there are $b, c \subseteq[0, k]$ such that $b \supseteq a, n<k, A \cap[0, k]=c, b \cap[0, n]=a$ and $|b|=|c|$. So $A=$ $(c \cup A / k) E_{0}^{\prime}(b \cup A / k) \in D_{b, k} \subseteq D_{a, n}$. And its class is dense.

Proposition 15. Let $\boldsymbol{E}$ be a meagre equivalence relation on $2^{\mathbb{N}}$ containing $E_{0}^{\prime}$. Then $E_{0} \leq_{\mathrm{B}} \boldsymbol{E}$.

Proof. Let $\left(D_{n}\right)$ be a decreasing sequence of dense open sets, such that $\boldsymbol{E} \cap \bigcap_{n} D_{n}=\emptyset$.

We will inductively construct sequences $b_{0}^{n}, b_{1}^{n} \in 2^{<\mathbb{N}}$ for $n \in \mathbb{N}$ such that for all $n,\left|b_{0}^{n}\right|=\left|b_{1}^{n}\right|, \overline{\overline{b_{0}^{n}}}=\overline{\overline{b_{1}^{n}}}:=\#\left\{k \mid b_{1}^{n}(k)=1\right\}$. And if $a_{s}:=$ $\left.b_{s(0)}^{0}\right\urcorner \cdots \frown b_{s(|s|-1)}^{|s|-1}$ for all $s \in 2^{<\mathbb{N}}$, then for any $s, t \in 2^{n}, N_{a_{s\urcorner 0}} \times N_{a_{t \wedge 1}}$ $\subseteq D_{n+1}$.

Suppose that this can be done. Then define $\alpha \mapsto \bigcup_{n} a_{\alpha \mid n}=a_{\alpha}$. This is clearly continuous. If now $\neg \alpha E_{0} \beta$, then for infinitely many $n, \alpha(n) \neq \beta(n)$. So for these $n,\left(a_{\alpha}, a_{\beta}\right) \in N_{a_{\alpha \uparrow n+1}} \times N_{a_{\beta \uparrow n+1}} \subseteq D_{n+1}$, which implies that $\left(a_{\alpha}, a_{\beta}\right) \in \bigcap_{k} D_{k} \subseteq \complement \boldsymbol{E}$.

Conversely, if $\alpha E_{0} \beta$, then for some $N$ we have $\forall n \geq N \alpha(n)=\beta(n)$. But then easily $\left.\left.a_{\alpha}=a_{\alpha \uparrow N}\right\urcorner b_{\alpha(N)}^{N}\right\urcorner b_{\alpha(N+1)}^{N+1} \cdots$ and $\left.\left.a_{\beta}=a_{\beta \uparrow N}\right\urcorner b_{\alpha(N)}^{N}\right\urcorner b_{\alpha(N+1)}^{N+1} \cdots$, so by the construction, $a_{\alpha} E_{0}^{\prime} \beta$. 
Now for the construction: Suppose that $b_{0}^{n}, b_{1}^{n}$ have been chosen for all $m<n$, enumerate $2^{n} \times 2^{n}$ by $\left(s_{0}, t_{0}\right), \ldots,\left(s_{k}, t_{k}\right)$ and take $c_{0}^{0}, c_{1}^{0} \in 2^{<\mathbb{N}}$ such that $N_{a_{s_{0}} \wedge c_{0}^{0}} \times N_{a_{t_{0}}} \wedge c_{1}^{0} \subseteq D_{n}$. This can be done as $D_{n}$ is dense and open in the product.

Extend $c_{0}^{0}, c_{1}^{0}$ to $c_{0}^{1}, c_{1}^{1}$ respectively in such a way that $N_{\left.a_{s_{1}}\right\urcorner c_{0}^{1}} \times N_{\left.a_{t_{1}}\right\urcorner c_{1}^{1}}$ $\subseteq D_{n}$

Again, extend $c_{0}^{1}, c_{1}^{1}$ to $c_{0}^{2}, c_{1}^{2}$ respectively in such a way that $N_{a_{s_{2}}} \neg c_{0}^{2} \times$ $N_{a_{t_{2}} \frown c_{1}^{2}} \subseteq D_{n}$, etc.

Finally, extend $c_{0}^{k}, c_{1}^{k}$ to $b_{0}^{n}, b_{1}^{n}$ respectively, so that $\left|b_{0}^{n}\right|=\left|b_{1}^{n}\right|, \overline{\overline{b_{0}^{n}}}=\overline{\overline{b_{1}^{n}}}$. This finishes the construction.

For the following, we recall that $\forall^{*} x R(x)$ means that the set $\{x \mid R(x)\}$ is comeagre, where $x$ varies over some Polish space.

THEOREM 16. Let $\boldsymbol{E}$ be an analytic equivalence relation on $[\mathbb{N}]^{\mathbb{N}}$ such that $E_{0}^{\prime} \subseteq \boldsymbol{E}$, i.e., $\boldsymbol{E}$ is $E_{0}^{\prime}$-invariant. Then either $E_{0} \leq_{\mathrm{c}} \boldsymbol{E}$ or there is some $A \in[\mathbb{N}]^{\mathbb{N}}$ such that $\boldsymbol{E}$ only has one class on $[A]^{\mathbb{N}}$.

Proof. By Corollary 3.5 of [15], if $E_{0} \mathbb{Z}_{\mathrm{c}} \boldsymbol{E}$, then $\boldsymbol{E}$ will be a decreasing intersection of $\aleph_{1}$ smooth equivalence relations:

$$
\boldsymbol{E}=\bigcap_{\omega_{1}} \boldsymbol{E}_{\xi}, \quad \boldsymbol{E}_{\xi} \subseteq \boldsymbol{E}_{\eta}, \quad \eta<\xi<\omega_{1} .
$$

Let $f_{\xi}:[\mathbb{N}]^{\mathbb{N}} \rightarrow \mathbb{R}$ be a Borel reduction of $\boldsymbol{E}_{\xi}$ to identity on $\mathbb{R}$. Then for any $A \in[\mathbb{N}]^{\mathbb{N}}$, there is a $B \in[A]^{\mathbb{N}}$ such that $f_{\xi}\left\lceil[B]^{\mathbb{N}}\right.$ is continuous. But since there is a dense $\boldsymbol{E}_{\xi}$-class the function has to be constant, that is, there is only one class.

We construct inductively a $\subseteq{ }^{*}$-decreasing sequence $\left(A_{\xi}\right)_{\omega_{1}}$ of infinite subsets of $\mathbb{N}$, with each $A_{\xi}$ being homogeneous for $\boldsymbol{E}_{\xi}$. Under $\mathrm{MA}+\neg \mathrm{CH}$ such a sequence can be diagonalised to produce an infinite $A_{\infty} \subsetneq^{*} A_{\xi}$ for all $\xi<\omega_{1}$. Now as $A_{\infty} \subsetneq^{*} A_{\xi}$ it is easily seen that $A_{\infty}$ is $E_{0}^{\prime}$-equivalent with some subset of $A_{\xi}$ and therefore also $\boldsymbol{E}_{\xi}$-equivalent with $A_{\xi}$ itself. Furthermore, the same holds for any infinite subset of $A_{\infty}$, so $A_{\infty}$ is homogeneous for all of the $\boldsymbol{E}_{\xi}$ and therefore for $\boldsymbol{E}$ as well.

As before, one sees that the property of having a homogeneous set is $\Sigma_{2}^{1}$, so we need only check that continuously reducing $E_{0}$ is $\Sigma_{2}^{1}$. For this it is easier to work with $\widetilde{E}_{0}$ as defined on $2^{\mathbb{N}}$ by $\alpha \widetilde{E}_{0} \beta \Leftrightarrow \exists n \forall m \geq n \alpha_{m}=\beta_{m}$. This is easily seen to be continuously bireducible with $E_{0}$, and moreover, it has the advantage that its restriction to any dense $G_{\delta}$ subset of $2^{\mathbb{N}}$ is again continuously bireducible with $E_{0}$. So the property of reducing $E_{0}$ (or equivalently $\widetilde{E}_{0}$ ) can be written as:

$\exists f: 2^{\mathbb{N}} \rightarrow[\mathbb{N}]^{\mathbb{N}}$ continuous

$$
\left[\forall^{*} \alpha \in 2^{\mathbb{N}} \forall \beta \in[\alpha]_{\widetilde{E}_{0}}(f(\alpha) \boldsymbol{E} f(\beta)) \& \forall \alpha, \beta \in 2^{\mathbb{N}}\left(\alpha \widetilde{E}_{0} \beta \vee \neg f(\alpha) \boldsymbol{E} f(\beta)\right)\right]
$$


So as the quantifier $\forall \beta \in[\alpha]_{\widetilde{E}_{0}}$ is over a countable set and the category quantifier $\forall^{*}$ preserves analyticity (see Theorem (29.22) in [17]), the statement is $\Sigma_{2}^{1}$.

4. Applications to Banach space theory. To avoid trivialities, let us suppose that all Banach spaces considered in this section are separable and infinite-dimensional.

In the course of the solution of the homogeneous space problem W. T. Gowers showed the following amazing result about the structure of subspaces of a Banach space: If $X$ is a Banach space, then it contains either an unconditional basic sequence or an H.I. subspace [10].

Here an H.I. (hereditarily indecomposable) space $Y$ is one in which no two infinite-dimensional subspaces form a direct sum. This property, which passes to subspaces, ensures that $Y$ cannot be isomorphic to any of its subspaces and cannot contain any unconditional basic sequence. Therefore, in the classification of the subspaces of a Banach space one can always suppose to be dealing with an H.I. space or a space with an unconditional basis.

Proposition 17. Let $\left(e_{i}\right)$ be a basic sequence in a Banach space. Then either $E_{0}$ Borel reduces to isomorphism of spaces spanned by subsequences of the basis or there is some infinite $A \subseteq \mathbb{N}$ such that $\left[e_{i}\right]_{A} \cong\left[e_{i}\right]_{B}$ for any infinite $B \subseteq A$.

Proof. Define the following equivalence relation on $[\mathbb{N}]^{\mathbb{N}}$ :

$$
A \cong B \Leftrightarrow\left[e_{i}\right]_{A} \cong\left[e_{i}\right]_{B} .
$$

Then $\cong$ is analytic and extends $E_{0}^{\prime}$. For suppose that $A E_{0}^{\prime} B$. Then $\left[e_{i}\right]_{A}$ and $\left[e_{i}\right]_{B}$ are spaces of the same finite codimension in $\left[e_{i}\right]_{A \cup B}$ and are therefore isomorphic. So the result follows from Theorem 16.

EXAMPLE 18 (Hereditarily indecomposable spaces). Suppose that a hereditarily indecomposable space $X$ is given. Then, as any Banach space contains a (conditional) basic sequence, we can suppose that $X$ has a basis $\left(e_{i}\right)$. By Proposition 17 , if $E_{0}$ does not reduce, there is a subsequence spanning a space isomorphic to some proper subspace, which contradicts the properties of H.I. spaces. So $E_{0}$ reduces to isomorphism of its subspaces. On the other hand, Theorem 1 shows that it has a continuum of incomparable subspaces, since it obviously cannot contain a minimal subspace. We can also mention a simple way to see that any H.I. space contains a continuum of non-isomorphic spaces: it is rather easy to see that there is a family $\left(A_{r}\right)_{r \in \mathbb{R}}$ of infinite subsets of $\mathbb{N}$ such that if $r<s$ then $A_{r} \subsetneq A_{s}$. So the spaces $\left[e_{i}\right]_{i \in A_{r}}$ are all isomorphic. 
THeOREM 19. Let $X$ be a Banach space such that the isomorphism relation between its subspaces does not reduce $E_{0}$. Then $X$ contains a reflexive subspace with an unconditional basis all of whose subsequences span isomorphic spaces.

Proof. Suppose that $E_{0}$ does not reduce to isomorphism between subspaces of $X$. Then using Gowers's dichotomy we can find a subspace $Y$ with an unconditional basis. Now, a recent result due to Ferenczi and Galego [7] says that $E_{0}$ Borel reduces to the isomorphism relation between subspaces of $c_{0}$ and $\ell_{1}$. So by James's characterisation of reflexivity, this basis must span a reflexive space, and by applying Proposition 17, we have our result.

Let us notice that if a basis $\left(e_{i}\right)$ has the property that no two disjointly supported block basic sequences are equivalent, then one can show that it satisfies

$$
\left(e_{i}\right)_{A} \approx\left(e_{i}\right)_{B} \Leftrightarrow\left[e_{i}\right]_{A} \cong\left[e_{i}\right]_{B} \Leftrightarrow A E_{0}^{\prime} B .
$$

See the work of Gowers and Maurey [12] for unconditional examples of such bases. An important precursor of their spaces can also be found in the article [2] by S. F. Bellenot. Therefore, as $E_{0}^{\prime}$ and $E_{0}$ are Borel bireducible, there are bases on which both equivalence and isomorphism between subsequences are exactly of complexity $E_{0}$.

We will now see an extension of some results by Ferenczi and the author [8], and Kalton [16].

THEOREM 20. Let $\left(e_{i}\right)$ be an unconditional basic sequence. Then either $E_{0}$ Borel reduces to isomorphism of spaces spanned by subsequences of the basis, or any space spanned by a subsequence is isomorphic to its square and its hyperplanes. And there is some infinite $A \subseteq \mathbb{N}$ such that for any infinite $B \subseteq A,\left[e_{i}\right]_{A} \cong\left[e_{i}\right]_{B}$.

Proof. Define the relation $\cong$ on $[\mathbb{N}]^{\mathbb{N}}$ as in the proof of Proposition 17 . By Proposition 15 , one sees that if $E_{0} \mathbb{Z}_{\mathrm{B}} \cong$, then $\cong$ must be non-meagre and therefore by Kuratowski-Ulam have a non-meagre class, which again by Lemma 14 is comeagre. So we can suppose we have some comeagre class $\mathcal{A} \subseteq[\mathbb{N}]^{\mathbb{N}}$. Seeing $[\mathbb{N}]^{\mathbb{N}}$ as a comeagre subspace of $2^{\mathbb{N}}, \mathcal{A}$ is also comeagre in $2^{\mathbb{N}}$. There is therefore a partition $A_{0}, A_{1}$ of $\mathbb{N}$ and subsets $B_{0} \subseteq A_{0}, B_{1} \subseteq$ $A_{1}$ such that for any $C \subseteq \mathbb{N}$, if $C \cap A_{0}=B_{0}$ or $C \cap A_{1}=B_{1}$, then $C \in \mathcal{A}$. In particular, $B_{0}, B_{1}, B_{0} \cup B_{1} \in \mathcal{A}$. Moreover, as the complement operation is a homeomorphism of $2^{\mathbb{N}}$ with itself, there is some $C$ such that $C, \complement C \in \mathcal{A}$. So identifying subsets of $\mathbb{N}$ with the Banach spaces they generate and using the fact that the basis is unconditional, and therefore that disjoint subsets form direct sums, we can calculate:

$$
\mathbb{N}=C \cup \complement C \cong C \oplus \complement C \cong B_{0} \oplus B_{0} \cong B_{0} \oplus B_{1} \cong B_{0} \cup B_{1} \cong B_{0} .
$$


So $\mathbb{N} \in \mathcal{A}$ and $\mathcal{A}$ consists of spaces isomorphic to their squares. Now for any $D \subseteq \mathbb{N}$,

$\mathbb{N} \oplus D \cong B_{0} \oplus B_{1} \oplus D \cong\left[B_{0} \cup\left(D \cap A_{1}\right)\right] \oplus\left[B_{1} \cup\left(D \cap A_{0}\right)\right] \cong \mathbb{N} \oplus \mathbb{N} \cong \mathbb{N}$.

This in particular shows that $\left[e_{i}\right]_{\mathbb{N}}$ is isomorphic to its hyperplanes.

We now notice that the argument is quite general, in the sense that we could have begun from any $\left[e_{i}\right]_{A}$ instead of $\left[e_{i}\right]_{\mathbb{N}}$, and therefore the results hold for any space spanned by a subsequence. The last statement of the theorem follows from Proposition 17.

Kalton [16] showed that if an unconditional basis only has a countable number of isomorphism classes on the subsequences of the basis, then the space spanned is isomorphic to its square and hyperplanes. The above result is along the same lines and we should mention that one can get uniformity results with a bit of extra care in the proof; see the article [8] by Ferenczi and the author for this.

Notice that permutative equivalence between subsequences of a basis $\left(e_{i}\right)$ induces an analytic equivalence relation on $[\mathbb{N}]^{\mathbb{N}}$ as follows:

$$
A \sim_{\mathrm{p}} B \Leftrightarrow\left(e_{i}\right)_{i \in A} \text { is permutatively equivalent to }\left(e_{i}\right)_{i \in B} .
$$

P. Casazza drew my attention to the following theorem from [4] (Proposition 6.2).

Theorem 21 (Bourgain, Casazza, Lindenstrauss, Tzafriri). If $\left(e_{i}\right)_{\mathbb{N}}$ is an unconditional basic sequence permutatively equivalent to all of its subsequences, then there is a permutation $\pi$ of $\mathbb{N}$ such that $\left(e_{\pi(i)}\right)_{\mathbb{N}}$ is subsymmetric.

Their statement of the theorem is slightly more general, but the general case is easily seen to follow from the infinite-dimensional Ramsey theorem of Galvin and Prikry (Theorem (19.11) in [17]).

Proposition 22. Let $\left(e_{i}\right)_{\mathbb{N}}$ be an unconditional basic sequence. Then either $E_{0}$ reduces to the relation of permutative equivalence of the subsequences of the basis, or there is some $B \in[\mathbb{N}]^{\mathbb{N}}$ such that $\left(e_{i}\right)_{i \in B}$ is subsymmetric.

Proof. Notice first that if $A E_{0}^{\prime} B$, then also $A \sim_{\mathrm{p}} B$. In other words, the relation $\sim_{\mathrm{p}}$ on $[\mathbb{N}]^{\mathbb{N}}$ is $E_{0}^{\prime}$-invariant. So if $E_{0}$ does not Borel reduce to $\sim_{\mathrm{p}}$, then by Theorem 16 there is some $A \in[\mathbb{N}]^{\mathbb{N}}$ such that $A \sim_{\mathrm{p}} B$ for all $B \in[A]^{\mathbb{N}}$. List $A$ increasingly as $a_{0}<a_{1}<a_{2}<\cdots$. Then

$$
e_{a_{0}}, e_{a_{1}}, e_{a_{2}}, \ldots
$$

is permutatively equivalent to all of its subsequences, whence by Theorem 21 there is some permutation $\pi$ of $A$ such that

$$
e_{\pi\left(a_{0}\right)}, e_{\pi\left(a_{1}\right)}, e_{\pi\left(a_{2}\right)}, \ldots
$$

is subsymmetric. 
Choose now some strictly increasing sequence $n_{0}<n_{1}<n_{2}<\cdots$ of elements of $A$ such that also

$$
\pi\left(n_{0}\right)<\pi\left(n_{1}\right)<\pi\left(n_{2}\right)<\cdots .
$$

Then

$$
e_{\pi\left(n_{0}\right)}, e_{\pi\left(n_{1}\right)}, e_{\pi\left(n_{2}\right)}, \ldots
$$

is a subsequence of $e_{\pi\left(a_{0}\right)}, e_{\pi\left(a_{1}\right)}, e_{\pi\left(a_{2}\right)}, \ldots$, and hence subsymmetric. Moreover, the set $B=\left\{\pi\left(n_{0}\right), \pi\left(n_{1}\right), \pi\left(n_{2}\right), \ldots\right\}$ is increasingly enumerated, so $\left(e_{i}\right)_{i \in B}$ is subsymmetric.

In connection with the above results, the reviewer drew my attention to the following result of Bourgain, Rosenthal and Schechtman and the question it naturally poses:

Theorem 23 ([5]). For $1<p<\infty, p \neq 2$, there are at least $\aleph_{1}$ nonisomorphic complemented subspaces of $L_{p}$.

Obviously, this result is not satisfactory, as one should expect a continuum of such subspaces. Can the methods used in this paper say something about this problem?

Acknowledgements. This article was written while the author was a doctoral student in Paris under the direction of Alain Louveau. I am sincerely grateful for all his help and the interest he showed in my work. Many thanks are also due to Valentin Ferenczi for friendship and numerous conversations on the subject. Finally, I am grateful to the reviewer for extensive comments, substantially improving the presentation of this paper.

Appendix. For the convenience of the reader, we include the proof that having a minimal subspace is $\Sigma_{2}^{1}$. This is a slightly amended version of the proof in [9] showing that having a block-minimal subspace is a $\Sigma_{2}^{1}$ property.

So suppose that $X$ has a minimal subspace and that it does not contain $c_{0}$. Then it has a minimal subspace with a basis $\left(e_{i}\right)$ and positively separated inevitable sets $F_{0}, F_{1}$ in the unit sphere of $\left[e_{i}\right]$. (Again this follows from the results of Odell and Schlumprecht [20,21].)

We let $\mathbb{D}=\left\{Y=\left(y_{i}\right) \in \operatorname{bb}_{\mathbb{Q}}\left(e_{i}\right) \mid \forall i y_{i} \in F_{0} \cup F_{1}\right\}$ and as in Lemma 7 let $\alpha(Y) \in 2^{\mathbb{N}}$ be defined by $\alpha(Y)(i)=0 \leftrightarrow y_{i} \in F_{0}$. Again $\mathbb{D}$ is large in $[Z]$ for any $Z \in \operatorname{bb}_{\mathbb{Q}}\left(e_{i}\right)$. Furthermore, let $\beta: 2^{\mathbb{N}} \leftrightarrow\left(\mathbb{Q}_{*}^{<\mathbb{N}}\right)^{\mathbb{N}}$ be some fixed recursive isomorphism.

Given a $Y=\left(y_{i}\right) \in \operatorname{bb}_{\mathbb{Q}}\left(e_{i}\right)$, any element $\left(\underline{\lambda}_{i}\right) \in\left(\mathbb{Q}_{*}^{<\mathbb{N}}\right)^{\mathbb{N}}$ codes a unique infinite sequence of block vectors (not necessarily consecutive) of $Y$, which we denote by $\left(\underline{\lambda}_{i}\right) \times Y$. So due to the minimality of $\left[e_{i}\right]$ there is for any $Y=\left(y_{i}\right) \in \operatorname{bb}_{\mathbb{Q}}\left(e_{i}\right)$ some $\left(\underline{\lambda}_{i}\right) \in\left(\mathbb{Q}_{*}^{<\mathbb{N}}\right)^{\mathbb{N}}$ such that $\left(e_{i}\right) \approx\left(\underline{\lambda}_{i}\right) \times Y$ (a standard 
perturbation argument shows that the basic sequence $\left(e_{i}\right)$ always embeds as a sequence of finite rational blocks, though not necessarily consecutive).

Set $\mathbb{W}=\left\{Y=\left(y_{i}\right) \in \operatorname{bb}_{\mathbb{Q}}\left(e_{i}\right) \mid\left(y_{2 i}\right) \in \mathbb{D} \wedge\left(e_{i}\right) \approx \beta \circ \alpha\left(y_{2 i}\right) \times\left(y_{2 i+1}\right)\right\}$. This is a Borel subset of $\operatorname{bb}_{\mathbb{Q}}\left(e_{i}\right)$.

We claim that $\mathbb{W}$ is large in $\operatorname{bb}_{\mathbb{Q}}\left(e_{i}\right)$. For suppose that $Z \in \mathrm{bb}_{\mathbb{Q}}\left(e_{i}\right)$ is given. Take some $V=\left(v_{i}\right) \in \mathbb{D} \cap[Z]$ and a $\left(\underline{\lambda}_{i}\right) \in\left(\mathbb{Q}_{*}^{<\mathbb{N}}\right)^{\mathbb{N}}$ such that $\left(e_{i}\right) \approx$ $\left(\underline{\lambda}_{i}\right) \times\left(v_{3 i+2}\right)$. Choose $y_{2 i}=v_{3 i}$ or $y_{2 i}=v_{3 i+1}$ such that $\beta \circ \alpha\left(y_{2 i}\right)=\left(\underline{\lambda}_{i}\right)$ and put $y_{2 i+1}=v_{3 i+2}$. Then obviously $Y \leq V \leq Z$ and $Y \in \mathbb{D}$.

So by Gowers's theorem [10], for any $\Delta>0$ there is a winning strategy $\tau$ for II for producing blocks in $\mathbb{W}_{\Delta}$ in some $Y=\left(y_{i}\right) \leq\left(e_{i}\right)$. If $\Delta=\left(\delta_{n}\right)$ is chosen small enough such that $\delta_{n}<\frac{1}{2} d\left(F_{0}, F_{1}\right)$ for all $n$, then we can modify $\tau$ a bit in such a way that the vectors of even index played by II are outright in $F_{0} \cup F_{1}$. So if $\Delta$ is chosen small enough, a perturbation argument shows that $\tau$ is in fact a strategy for playing blocks in $\mathbb{W}$.

This shows that if $X$ has a minimal subspace, but does not contain an isomorphic copy of $c_{0}$, then there are a basic sequence $\left(e_{i}\right)$, an element $Y \in \operatorname{bb}_{\mathbb{Q}}\left(e_{i}\right)$ and a continuous function $\Theta:[Y] \rightarrow \overline{\operatorname{span}}\left(e_{i}\right)^{\mathbb{N}}$, defined by

$$
\Theta(Z)=\beta \circ \alpha\left((\tau(Z))_{2 i}\right) \times\left((\tau(Z))_{2 i+1}\right)
$$

such that for all $Z \in[Y]$, we have $\overline{\operatorname{span}}(\Theta(Z)) \subseteq \overline{\operatorname{span}}(Z)$ and $\left(e_{i}\right) \approx \Theta(Z)$. On the other hand, containing a copy of $c_{0}$ is evidently a $\Sigma_{1}^{1}$ property, so the disjunction of the two becomes $\Sigma_{2}^{1}$.

\section{References}

[1] J. Bagaria and J. López-Abad, Weakly Ramsey sets in Banach spaces, Adv. Math. 160 (2001), 133-174.

[2] S. F. Bellenot, The Banach spaces of Maurey and Rosenthal and totally incomparable bases, J. Funct. Anal. 95 (1991), 96-105.

[3] B. Bossard, A coding of separable Banach spaces. Analytic and coanalytic families of Banach spaces, Fund. Math. 172 (2002), 117-152.

[4] J. Bourgain, P. G. Casazza, J. Lindenstrauss and L. Tzafriri, Banach spaces with a unique unconditional basis, up to permutation, Mem. Amer. Math. Soc. 54 (1985), no. 322 .

[5] J. Bourgain, H. P. Rosenthal and G. Schechtman, An ordinal $L^{p}$-index for Banach spaces, with application to complemented subspaces of $L^{p}$, Ann. of Math. (2) 114 (1981), 193-228.

[6] V. Ferenczi, Minimality, homogeneity and topological 0-1 laws for block-subspaces in a Banach space, preprint.

[7] V. Ferenczi and E. M. Galego, Equivalence relations which are Borel reducible to isomorphism between separable Banach spaces, preprint.

[8] V. Ferenczi and C. Rosendal, On the number of non-isomorphic subspaces of a Banach space, Studia Math., to appear.

[9] - - - Ergodic Banach spaces, Adv. Math., to appear. 
[10] W. T. Gowers, An infinite Ramsey theorem and some Banach-space dichotomies, Ann. of Math. (2) 156 (2002), 797-833.

[11] —, Recent results in the theory of infinite-dimensional Banach spaces, in: Proc. Internat. Congress of Mathematicians, Zürich, 1994, Vol. 2, Birkhäuser, Basel, 1995, 933-942.

[12] W. T. Gowers and B. Maurey, Banach spaces with small spaces of operators, Math. Ann. 307 (1997), 543-568.

[13] L. A. Harrington, A. S. Kechris and A. Louveau, A Glimm-Effros dichotomy for Borel equivalence relations, J. Amer. Math. Soc. 3 (1990), 903-928.

[14] L. A. Harrington, D. Marker and S. Shelah, Borel orderings, Trans. Amer. Math. Soc. 310 (1988), 293-302.

[15] G. Hjorth and A. S. Kechris, Analytic equivalence relations and Ulm-type classifications, J. Symbolic Logic 60 (1995), 1273-1300.

[16] N. J. Kalton, A remark on Banach spaces isomorphic to their squares, in: Function Spaces (Edwardsville, IL, 1998), Contemp. Math. 232, Amer. Math. Soc., Providence, RI, 1999, 211-217.

[17] A. S. Kechris, Classical Descriptive Set Theory, Grad. Texts in Math. 156, Springer, New York, 1995.

[18] R. A. Komorowski and N. Tomczak-Jaegermann, Banach spaces without local unconditional structure, Israel J. Math. 89 (1995), 205-226; Erratum, ibid. 105 (1998), 85-92.

[19] J. López-Abad, Coding into Ramsey sets, CRM preprint 557 (2003).

[20] E. Odell and T. Schlumprecht, The distortion problem, Acta Math. 173 (1994), 259-281.

[21] - - - Distortion and asymptotic structure, in: Handbook of the Geometry of Banach Spaces, Vol. 2, North-Holland, Amsterdam, 2003, 1333-1360.

[22] S. Todorcevic, High-Dimensional Ramsey Theory and Geometry of Banach Spaces, forthcoming book.

Mathematics 253-37

California Institute of Technology

Pasadena, CA 91125, U.S.A.

E-mail: rosendal@ccr.jussieu.fr

Received 9 June 2004;

in revised form 2 December 2004 